\title{
Assessment of Sting Effect on X-31 Aircraft Model Using CFD
}

\author{
Adam Jirásek*and Russell M. Cummings ${ }^{\dagger}$ \\ United States Air Force Academy, USAFA, CO 80840, USA
}

\begin{abstract}
The article describes the computational evaluation of the effect a belly mounted sting on the aerodynamics characteristics of an X-31 wind tunnel model. The investigation includes results of steady and time-dependent CFD analysis of the X-31 model with and without sting. The values of the steady lift and pitching moment coefficients as well as the pitching moment of model undergoing a manuever are compared to each other and to the wind tunnel data. The results of this analysis show the detrimental effect of sting on stability of the vortical structure above the wing as well as a substantial increase of noise in pitching moment data.
\end{abstract}

\section{Nomenclature}

$\alpha$

$c_{M}$

$c_{N}$

$h_{i}$

$M$

$t$ angle of attack (deg)

pitching moment coefficient $(\mathrm{m} / \mathrm{gAC})$

normal force coefficient N/GA

Volterra kernels

Mach number v/a

time $(\mathrm{s})$

\section{Introduction}

$\mathrm{D}$ EFINING the Stability and Control (S\&C) characteristics of an airplane are probably one of the most difficult and expensive aspects of an aircraft development. The difficulties are partially due to the fact that the S\&C phase of design is extended to the very end of the development process, and sometimes even beyond it, causing occasionally unexpected and expensive twists along the project paths requiring changes on the aircraft. Since these can occur in the very late stages of the project, they are very expensive and often comes with detrimental effect to the expected performance. It is therefore of utmost importance to be able to predict $\mathrm{S} \& \mathrm{C}$ characteristics of the aircraft in the early stages of its development. One tool used to predict S\&C characteristics of an aircraft is a wind tunnel measurement. Wind tunnel testing is accurate and cheaper then flight test, however usually works in different limits of the physical space then real world situations - ie. different Reynolds number, different Strouhal number, smaller dimensions of the physical space biased by uncertainties in boundary conditions, etc. In addition, wind tunnel testing is often biased by the wind tunnel equipment necessary to keep the wind tunnel model in the wind tunnel test section.

One such example is an X-31 model tested in the DLR wind tunnel. ${ }^{1}$ The model is connected to the wind tunnel support by a belly mounted sting. The second setup includes a rear mounted sting. This article investigates the effect of a belly mounted sting on the aerodynamic characteristics of the X-31 wind tunnel model using time dependent CFD calculations. The effect is measured in terms of differences in the values of the lift and pitching moment of steady and maneuvering model as well as the amount of noise in the CFD data due to presence of the belly mounted sting.

\footnotetext{
${ }^{*}$ National Research Council, NRC, Research Associate, Department of Aeronautics, AIAA Member

$\dagger$ Professor of Aeronautics, Department of Aeronautics, AIAA Associate Fellow
} 


\section{Computational Models}

\section{A. Cobalt CFD code}

Cobalt $^{2}$ is a cell-centered, finite volume CFD code. It solves the unsteady, three-dimensional, compressible Reynolds Averaged Navier-Stokes (RANS) equations on hybrid unstructured grids. Its foundation is based on Godunov's first-order accurate, exact Riemann solver. Second-order spatial accuracy is obtained through a Least Squares Reconstruction. A Newton sub-iteration method is used in the solution of the system of equations to improve time accuracy of the point-implicit method. Strang et al. ${ }^{2}$ validated the numerical method on a number of problems, including the Spalart-Allmaras model, which forms the core for the Detached Eddy Simulation $(D E S)$ model available in Cobalt. Tomaro et al. ${ }^{3}$ converted the code from explicit to implicit, enabling CFL numbers as high as $C F L \approx 10^{6}$. Grismer et al. ${ }^{4}$ parallelized the code, with a demonstrated linear speed-up on as many as 4,000 processors. The parallel METIS (PARMETIS) domain decomposition library of Karypis et al. ${ }^{5}$ is also incorporated into Cobalt. New capabilities include rigid-body and 6 DOF motion, equilibrium air physics. An overset grid capability and a coupled aeroelastic simulation capability is also implemented. The code has been extensively used for S\&C analysis of a number of aircraft. ${ }^{6-10}$

The results of unsteady calculations were filter using low/high pass frequency filter. ${ }^{11}$

\section{X-31 aircraft model}

The X-31 $12-14$ is a typical case of an advanced fighter aircraft. It has been a subject of numerous flight tests ${ }^{15-19}$ wind tunnel tests ${ }^{20,21}$ and CFD. ${ }^{22,23}$ This test case has been provided to the partners participating in NATO RTO task group AVT-161 Assessment of Stability and Control Prediction Methods for NATO Air and Sea Vehicles. The objective of this task group is to evaluate CFD codes against wind tunnel data sets of two different aircraft configurations. A wind tunnel model used in experiments at DLR contains the canard, the LEX, the wind, the fuselage flap, the horizontal stabilizator and the rudder. ${ }^{21}$ The test setup does not consider any flow through the inlet. The wind tunnel model was equipped with the moving lift and control surfaces. Between each of the surface and the main body of the model were enabling mechanical movement of the surface. The effect of the gaps has been investigated in the wind tunnel ${ }^{21}$ and using CFD. ${ }^{23}$ It has been found that gaps substantially alter the flow-field above the wing giving rise to two co-rotating primary vortices. ${ }^{23}$ The values of the global lift, drag and momentum coefficients were, however, changed only mildly. Considering this mild dependence of the global forces/moments and in order to avoid having prohibitively large meshes used for unsteady CFD tests, the gaps in the model were sealed. The model is mounted in the wind tunnel using two setups. The first setup uses a belly mounted sting connecting the model to the support desk in the wind tunnel ceiling. The sting - model fuselage junction is located right under the main wing. This setup enables six degrees of freedom motions. The second setup uses an aft mounted sting connected to an arm in the wind tunnel.

\section{A. Computational Meshes}

The mesh generation process consists of two steps. In the first step, the inviscid tetrahedral mesh is generated using IcemCFD code. This mesh is then used as a background mesh in the second step in the mesh generator TRITET. ${ }^{24,25}$ TRITET first builds prism layer using frontal technique and then rebuilds the inviscid mesh while respecting size of the original inviscid mesh from IcemCFD. The mesh has nominally 18 prism layers with the stretching ratio of 1.18. The total number of cells is 13 million. Figure 1 shows the unstructured mesh around the X-31 geometry without and with the belly mounted sting.

\section{Steady Analysis}

The comparison of pitching moment shows the results of rhe steady analysis of the X-31 model with and without belly mounted sting using two different turbulence models - SA and SARC The effect of sting on the static values of lift is an offset of abou 0.1. There is, however, a very strong effect of the sting on pitching moment. The sting moves the moment by a constant offset of about 0.03 .

As shown in figure 2 this trend is reproduced by steady CFD calculations. The graph includes the results of steady analysis using two different turbulence models - SA and SARC. SARC model improves results

Distribution A, Approved for public release, distribution unlimited

2 of 10

American Institute of Aeronautics and Astronautics 


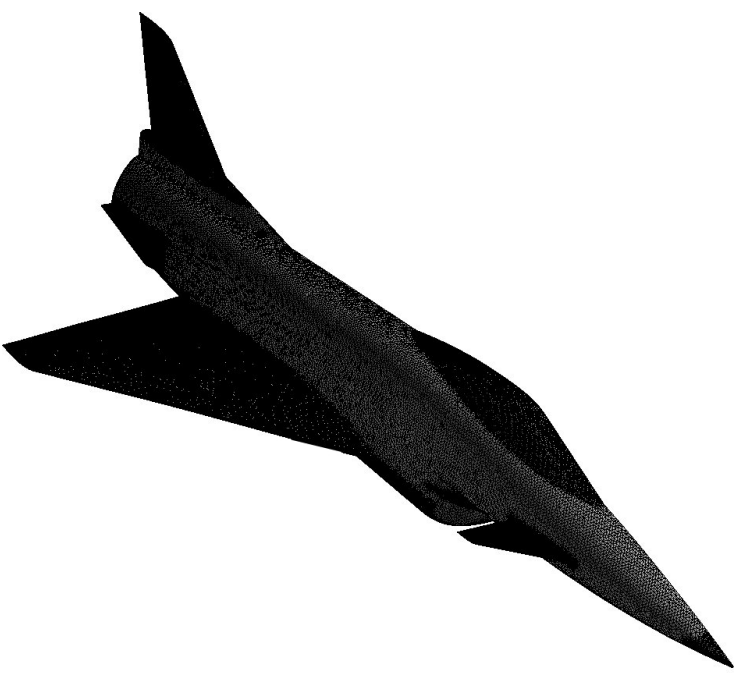

(a) Wind tunnel model without sting

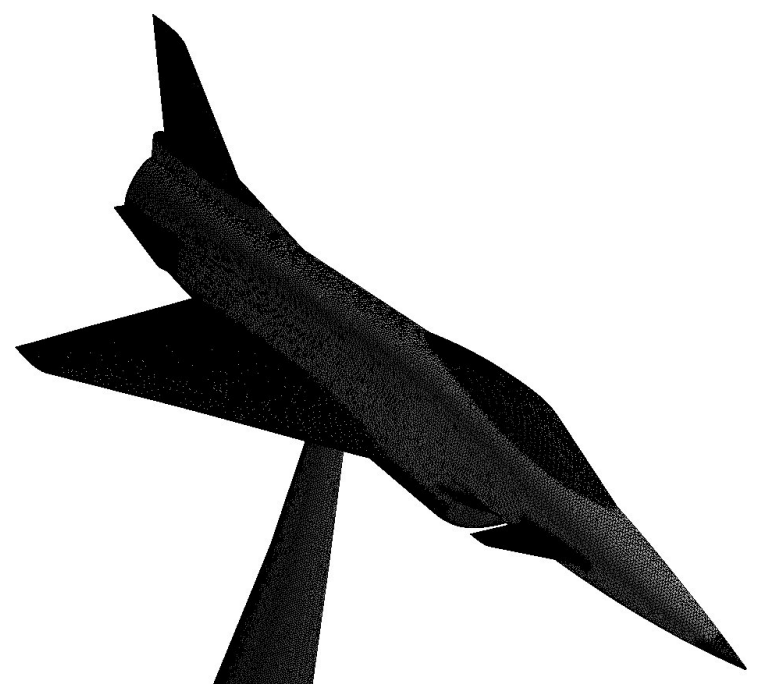

(b) Wind tunnel model with sting

Figure 1. Mesh around X-31 aircraft models

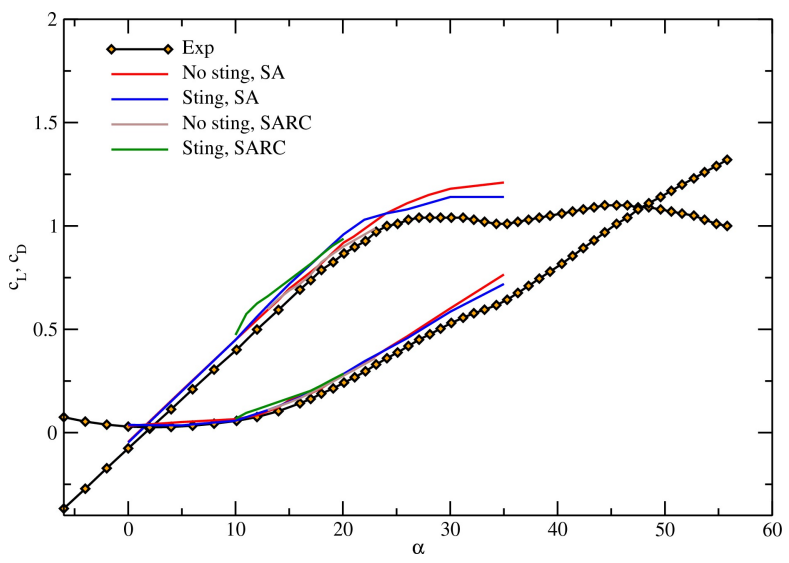

(a) $c_{L}, c_{D}$

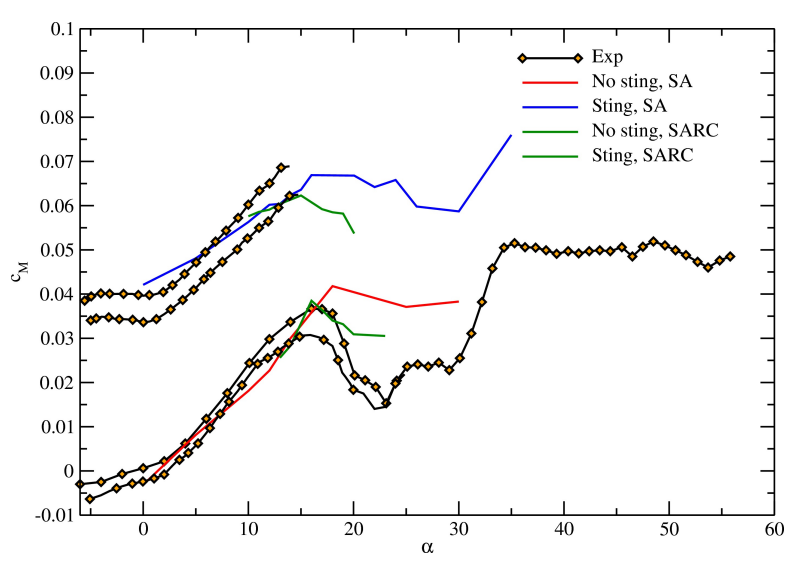

(b) $c_{M}$

Figure 2. Lift, drag and pitching moment, different turbulence models

Distribution A, Approved for public release, distribution unlimited

$$
3 \text { of } 10
$$


at angles above $\alpha=15 d g r$ indicating drop of pitching moment, however does not change character of the pitching moment curve of the model without and with sting.

\section{Unsteady Analysis}

\section{A. Flow Topology With Sting}

Figure 3 shows the the flow around X-31 at angle of attack $\alpha=20.06^{\circ}$ The flow has a vortical structure

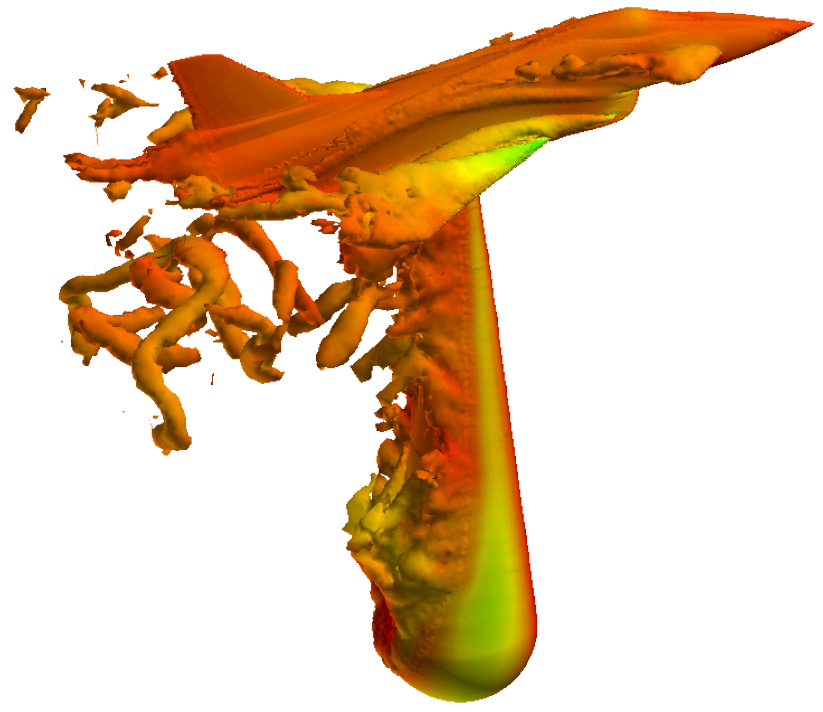

Figure 3. Vorticity around X-31 aircraft configuration

above the canard and main wing. Some of the smaller vortical structures arise from the place where the inlet cover is located. There is a wake structure behind the model sting. It is moving downstream and depending on the angle of incidence of entire model, it may colide with the aircraft surface.

Figure 4 shows Power Density Spectra (PDS) of the lift of the wing. The spectrum is rather noisy, however some important frequencies corresponding to certain features in the flow (see Cummings et al. ${ }^{26}$ for details) can be distinguished, mainly: Strouhal numbers in range of $S_{h} \approx 0.05$ and $S_{h} \approx 0.11$ corresponding to vortex breakdown, several distinctive peaks in range of $S_{h} \approx 0.2$ and $S_{h} \approx 0.4$ corresponding to vortex shedding at high $\alpha$ and $S_{h} \approx 1$ and $S_{h} \approx 2$ corresponding to Helical mode instability. Several peaks can be located above the value of Strouhal number $S_{h}>3$ possibly corresponding to Helical mode instability and shear layer instabilities.

\section{B. Noise in the Steady Data}

Figure 5 shows a time history of the normal and axial force and pitching moment coefficients of the wing, fuselage and of entire geometry. The angle of attack for this particular case were obtained at angle of attack $\alpha=0 d g r$. As can be seen, adding a belly mounted sting introduced a substantial amount of noise to both the forces and moment coefficient. As an example Figure 6 shows DFT of the pitching moment of the wing of the geometry without and with sting and of the sting itself. Rather flat spectrum of the normal force coefficient of the wing (Figure 6(a)) and fuselage (Figure 6(b)) of the geometry without sting is dramatically changed by adding the sting. Peaks in spectrum of the wing and fuselage of the geometry with sting is similar to the spectrum of the sting itself at frequencies up to $f=5 \mathrm{~Hz}$ suggesting that large, low frequency structures have profound effect on aerodynamics characteristics of at least the two major parts of aircraft.

\section{Noise due to model vibrations}

Another possible source of noise in the wind tunnel data are vibration of the model in the wind tunnel. The exact model position is measured by the optical positioning system.

In order to investigate an effect of the model vibration, we use the Volterra ROM developed in. ${ }^{27}$ Figure

Distribution A, Approved for public release, distribution unlimited

$$
4 \text { of } 10
$$

American Institute of Aeronautics and Astronautics 


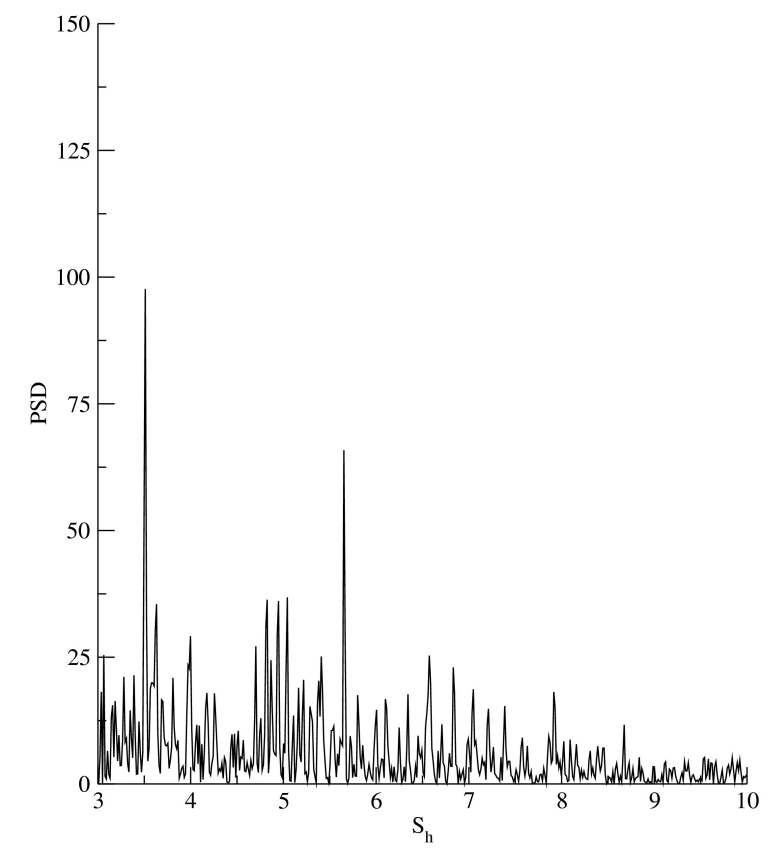

Figure 4. PDS of the wing and sting

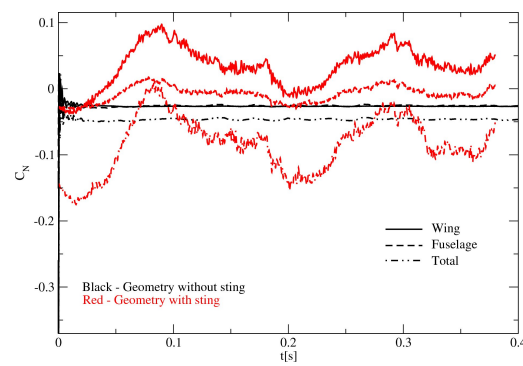

(a) $c_{N}$

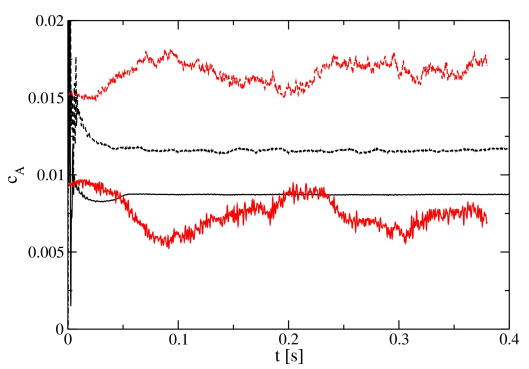

(b) $c_{A}$

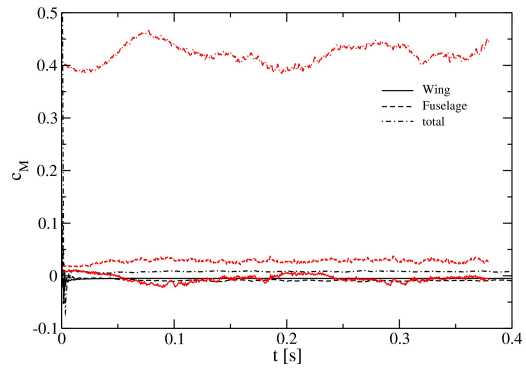

(c) $c_{M}$

Figure 5. Noise in normal force and pitching moment, X31

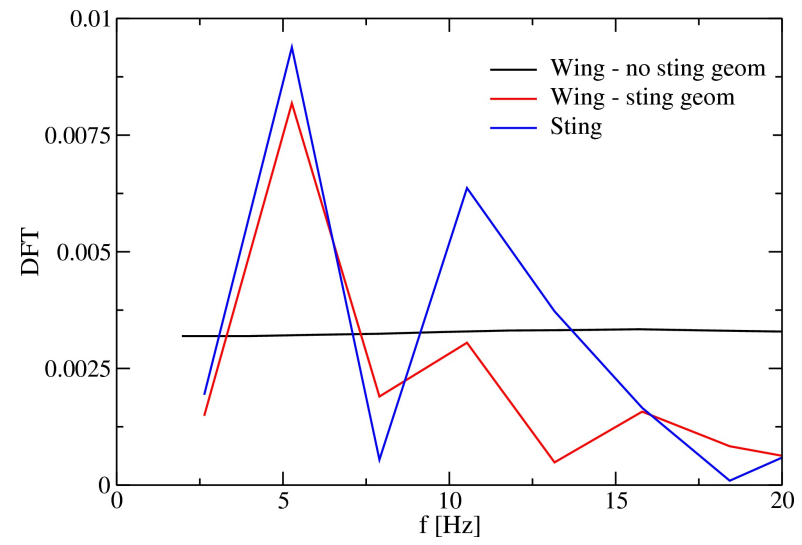

(a) Wing

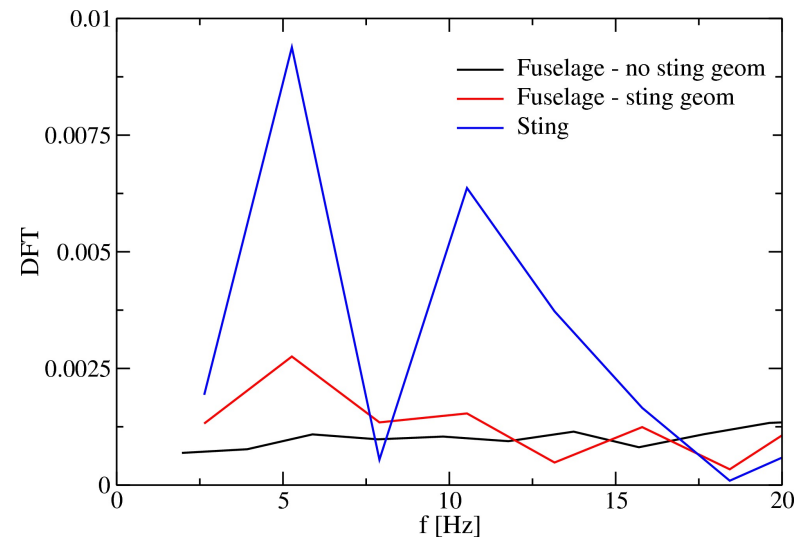

(b) Fuselage

Figure 6. DFT of the pitching moment on the wing, X31

Distribution A, Approved for public release, distribution unlimited

$$
5 \text { of } 10
$$


7 (a) shows the values of actual angle of attack measure in the wind tunnel. Figure 7(b) shows actual values of the pitching moment as a result of Volterra ROM. As can be seen the amount of noise in pitching moment

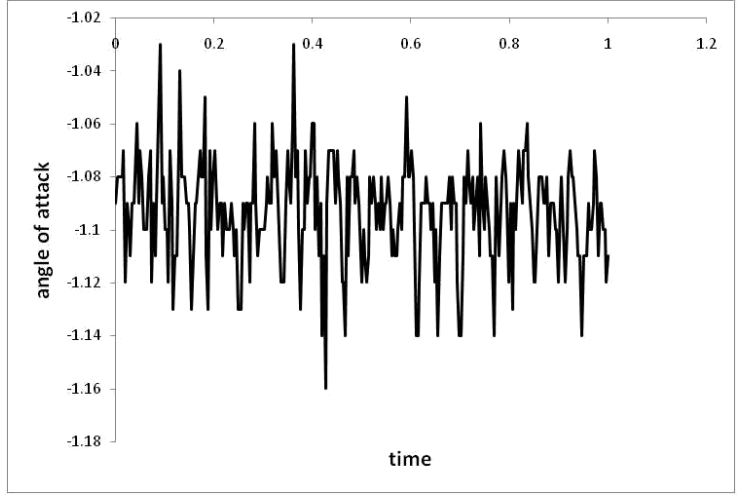

(a) Actual angle of attack

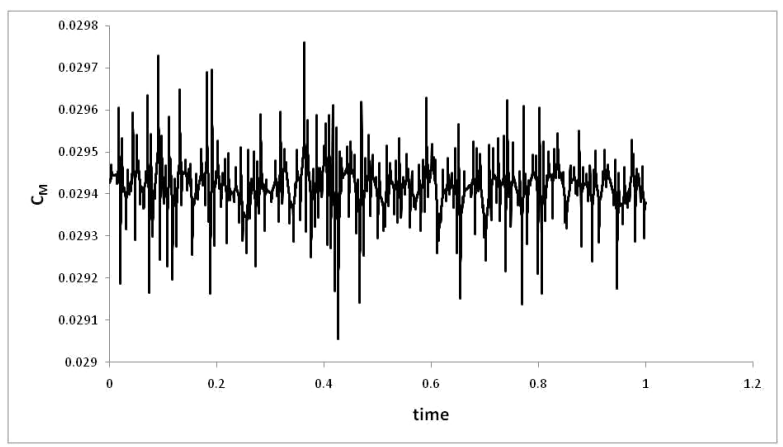

(b) Pitching moment

Figure 7. Low frequency data of the pitching moment

data due to model vibrations is very small and is of higher frequency then what we can see in the actual pitching moment data. It can be therefore concluded that model vibrations have negligible effect and does not have to be considered in the future CFD analysis.

\section{Dynamic Motion}

The dynamic motion used to investigate the effect of sting is a pitching motion denoted Sino4. Figure 8 shows angle of attack versus time. Figure 9 shows the comparisons of the CFD results and wind tunnel test.

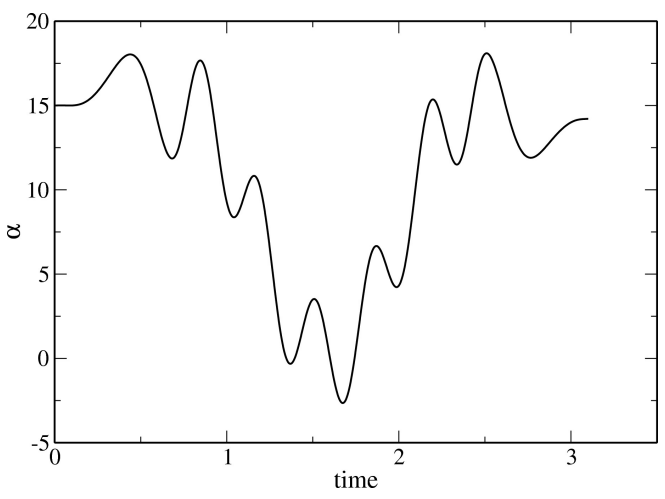

Figure 8. Angle of attack versus time, Sino4 motion

During the analysis, the data were split into the low and high-frequency part. Figure 10 shows comparison of the lift and pitching moment for CFD solution of both geometries to the wind tunnel data. In addition, the figure includes the results of linear Volterra ROM ${ }^{27}$ It is evident that the presence of the sting has detrimental effect on the stability of the vortical structure above the wing inducing premature vortex breakdown which occurs at angle of attack $15 \mathrm{deg}$ and results in larger pitching moment drop. Such tendency negatively effect predictability of any reduced order model built using this data set. It should be noted though, that this tendency is not seen in the wind tunnel test results which are obtained using the geometry with the sting. On the lift side, the presence of noise is relatively small for both geometries, the effect of sting is visible as a constant increase of lift $\Delta C_{L} \approx 0.1$.

Plotting the high-frequency component versus time as done in Figure 11 shows that adding sting to the model geometry gives rise to a larger amount of noise data at all angles which suggests that the biggest source of the noise is the wake behind the sting and its interaction with model. The amount of noise in result of model without sting geometry shown in figure 11(b) varies with angle of attack and thus can be

Distribution A, Approved for public release, distribution unlimited

$$
6 \text { of } 10
$$




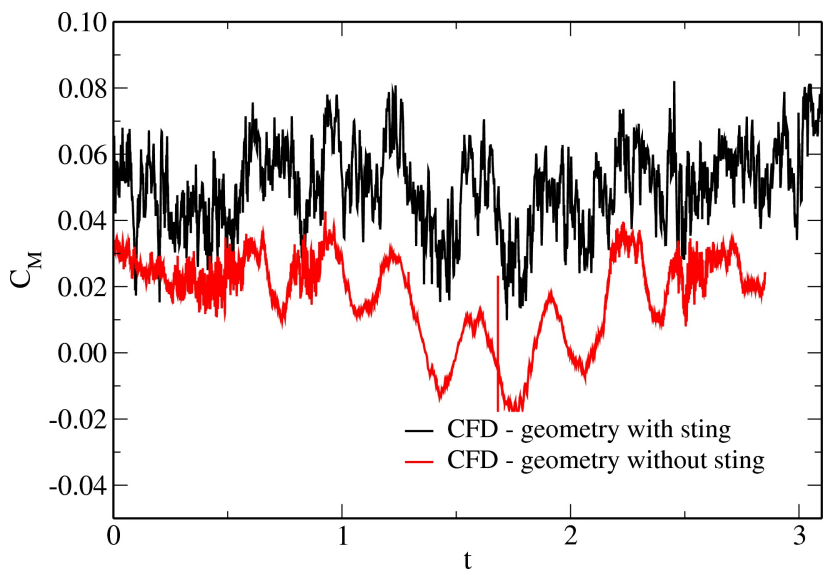

Figure 9. Pitching moment vs. time for X-31 with and without sting

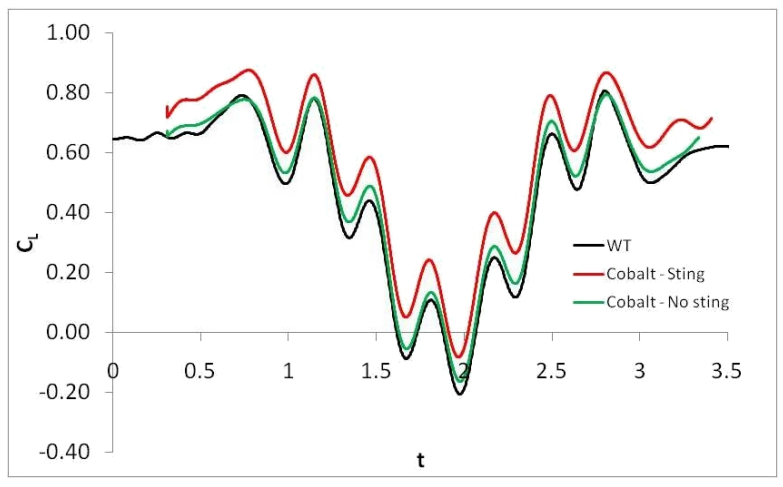

(a) Lift

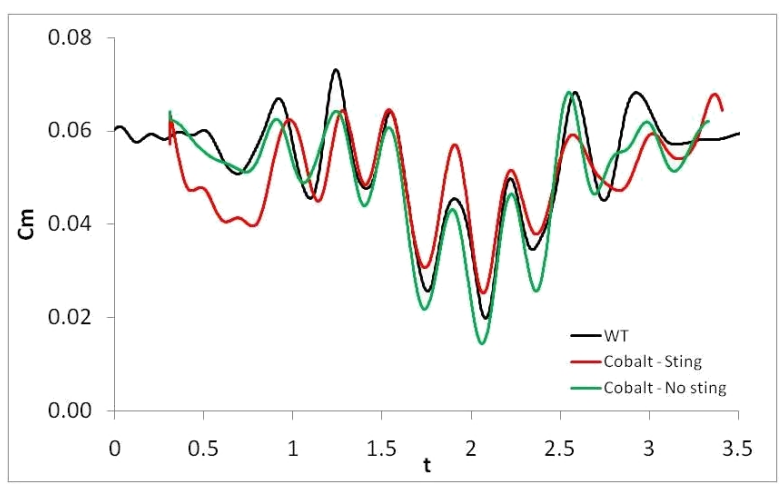

(b) Pitching moment

Figure 10. Low frequency data of the pitching moment

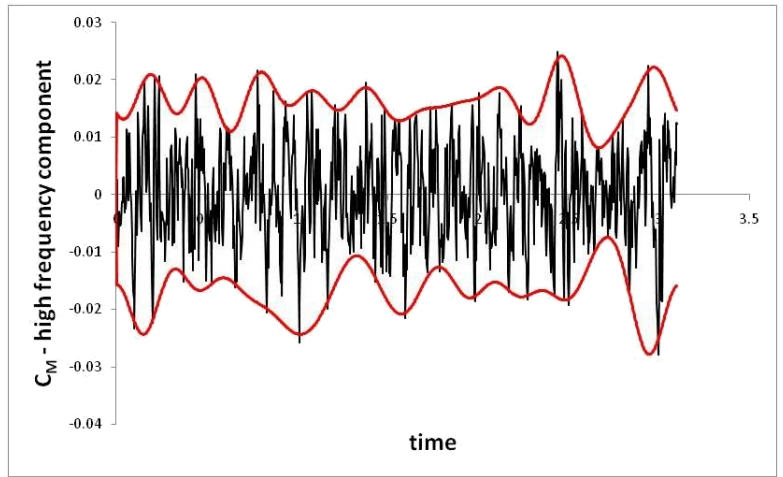

(a) Sting geometry

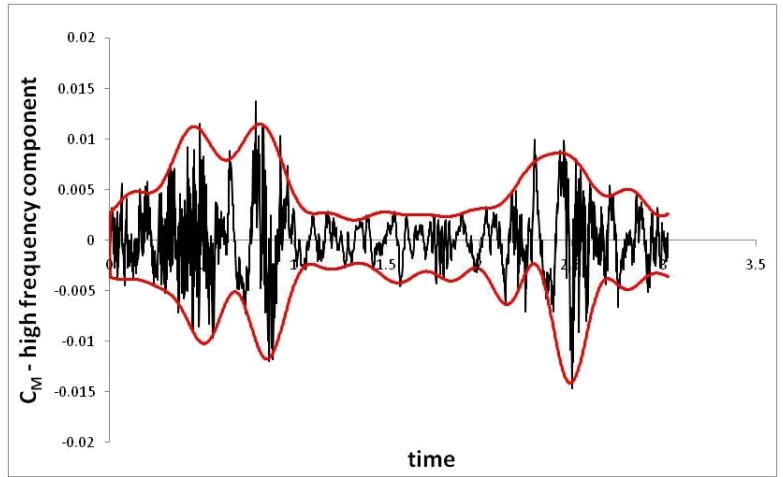

(b) No sting geometry

Figure 11. High-frequency component of the pitching moment

Distribution A, Approved for public release, distribution unlimited

$$
7 \text { of } 10
$$


directly attributed to increased amount of unsteadiness in the flow above wing after the vortex breaks down. In such case the measure of noise can be directly related to the instabilities of the flow above the wing where the vortical structure takes place.

\section{A. Analysis of the dynamic motion using SIDPAC}

The Sino4 motion was analyzed using System IDentification Program for AirCraft, SIDPAC code developed by Morelli. ${ }^{28,29}$ SIDPAC is a global nonlinear parameter modeling technique based on regression methods specifically for aircraft applications. This technique is used to estimate the functional relationship between the independent variables of the aircraft motion $(\alpha, \beta, p, q, r$, etc.) and the computed aerodynamic loads. The resulting SIDPAC model can then be used to interpolate aerodynamic loads within the parameter space. Both raw and filtered data were used for analysis. Figure 12 shows raw and filtered data set along with SIDPAC model for both geometries. The model for geometry with sting and raw CFD data set resulted in

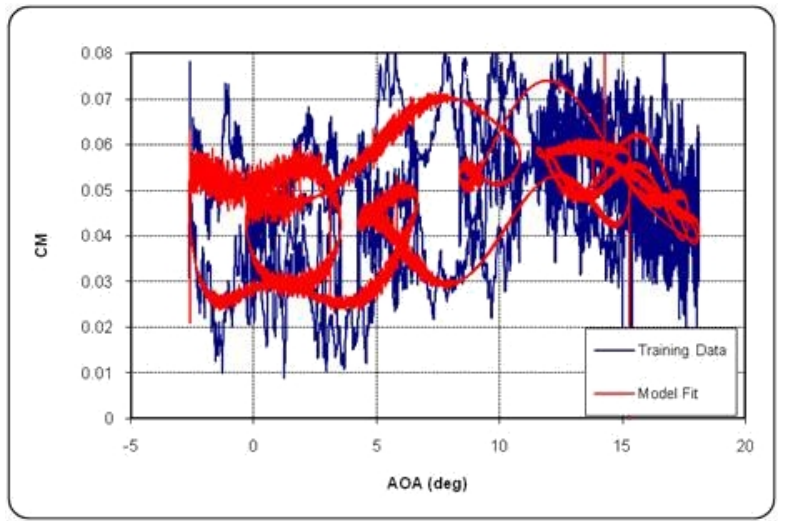

(a) Raw data - sting geometry

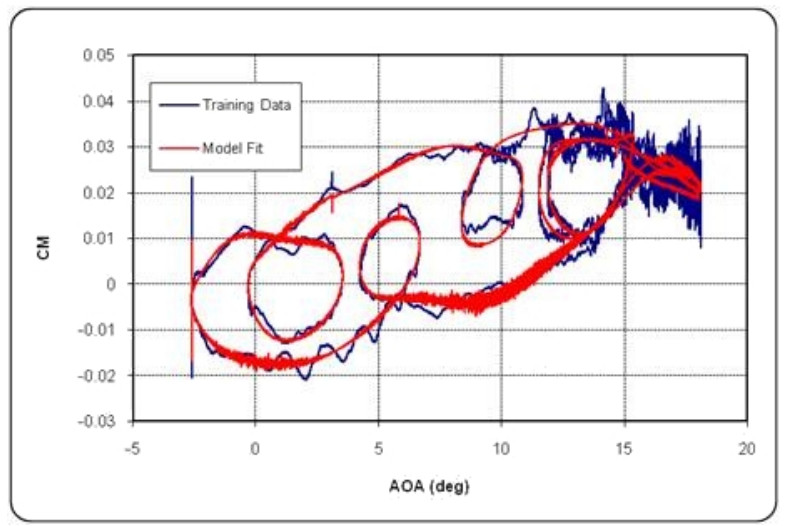

(c) Raw data - no sting geometry

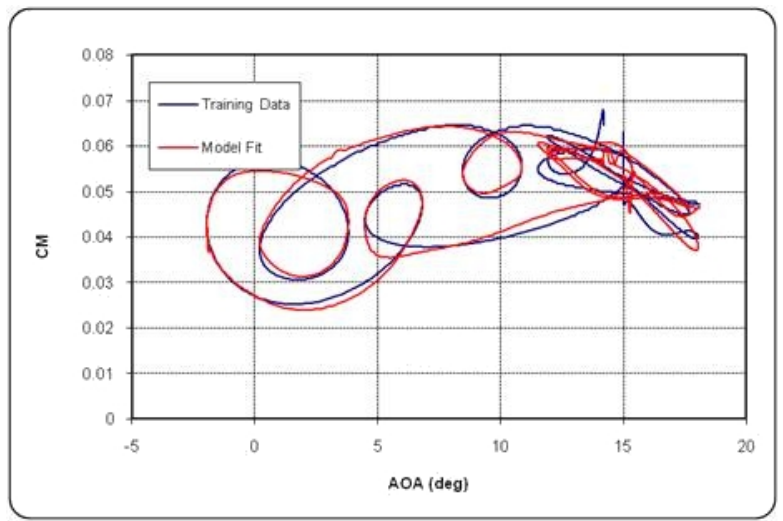

(b) Filtered data - sting geometry

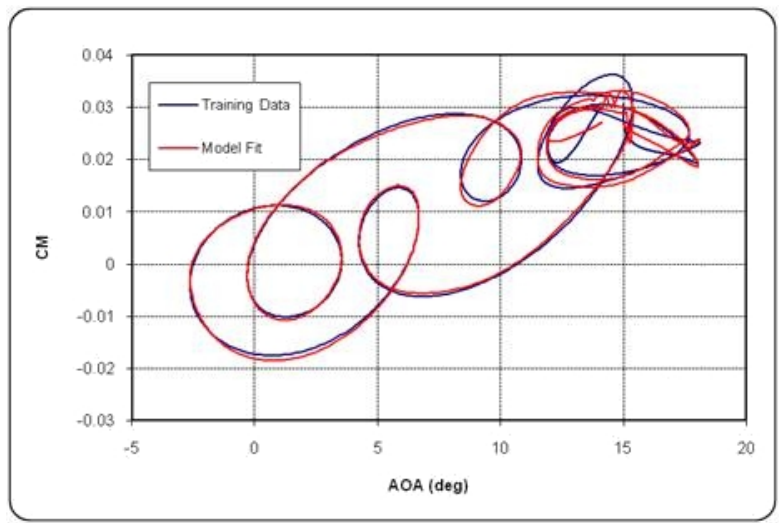

(d) Filtered data - no sting geometry

Figure 12. DATA and SIDPAC model comparisons

29 term with a final value of $R^{2}$ around $54 \%$. Adding other terms did not improve $R^{2}$. The SIDPAC analysis of the sting geometry with CFD data filtered prior to use in SIDPAC resulted in a model having 12 term and $R^{2}$ nearly $90 \%$. Comparing the four most important terms in both model, they are different.

The geometry without sting has considerably lower amount of noise in data producing a model with 38 terms and $R^{2}$ around $96 \%$ for raw data and 11 terms with $R^{2}$ almost $99 \%$ for filtered data. Comparing five the most important terms in the model shows the models have identical structure. Also, comparing figures 12(c) and 12 (d) shows the noise is present only around angle 15 which is the angle at which the vortex breakdown occurs. In this case, the presence of noise did not change substantially the outcome of the SIDPAC analysis.

Distribution A, Approved for public release, distribution unlimited 


\section{Conclusion}

This article investigates the effect of the sting on the aerodynamic characteristics of an X-31 wind tunnel model. Although inevitable, it was found that using sting as a mean of positioning the model in the wind tunnel plays a significant role on the pitching moment. It adds a constant offset to the lift and pitching moment data and is a source of a substantial amount of noise. In addition, the highly unsteady flow behind sting interferes with the flow around the model which, can, gives rise to premature vortex breakdown and stronger drop in pitching moment. It is suggested that future investigation does not consider the geometry of the sting.

\section{Acknowledgment}

The authors would like to thank the Air Force Office of Scientific Research, AFOSR, the United States Air Force Academy, USAFA - Modeling and Simulation Research Center and the National Research Council (NRC) for their generous financial support throughout this project. The Arctic Region Supercomputing Center is acknowledged for providing access to high performance computing facilities. The authors want to thank David McDaniel of University of Birmingham for help with SIDPAC code.

\section{References}

${ }^{1}$ Löser, T., "RTO/AVT-161: Assessment of Stability and Control Prediction Methods for NATO Air and Sea Vehicles Thomas Lser, DNW-NWB, X-31 Steady state and dynamic wind tunnel tests," NATO RTO AVT-113 presentation, May 2008.

${ }^{2}$ Strang, W. Z., Tomaro, R. F., and Grismer, M. J., "The Defining Methods of Cobalt: A Parallel, Implicit, Unstructured Euler/Navier- Stokes Flow Solver," AIAA Paper 99-0786, Jan. 1999.

${ }^{3}$ Tomaro, R. F., Strang, W. Z., and Sankar, L. N., "An implicit algorithm for solving time dependent flows on unstructured grids," AIAA Paper 1997-0333, Jan. 1997.

${ }^{4}$ Grismer, M. J., Strang, W. Z., Tomaro, R. F., and Witzemman, F. C., "Cobalt: A Parallel, Implicit, Unstructured Euler/Navier-Stokes Solver," Adv. Eng. Software, Vol. 29, No. 3-6, June 1998.

${ }^{5}$ Karypis, G., Schloegel, K., and Kumar, V., "Parmetis: Parallel Graph Partitioning and Sparse Matrix Ordering Library, Version 3.1," Technical report, Dept. Computer Science, University of Minnesota, 2003.

${ }^{6}$ Morton, S., McDaniel, D., and Cummings, R., "F-16XL Unsteady Simulations for the CAWAPI Facet of RTO Task Group AVT-113," AIAA-2007-493, 45th AIAA Aerospace Sciences Meeting and Exhibit, Reno, Nevada, Jan. 8-11, 2007.

${ }^{7}$ Görtz, S., McDaniel, D., and Morton, S., "Towards an Efficient Aircraft Stability and Control Analysis Capability Using High-Fidelity CFD," AIAA-2007-1053, 45th AIAA Aerospace Sciences Meeting and Exhibit, Reno, Nevada, Jan. 8-11, 2007.

${ }^{8}$ Dean, J., Morton, S., McDaniel, D., and Görtz, S., "Efficient High Resolution Modeling of Fighter Aircraft with Stores for Stability and Control Clearance," AIAA-2007-1652, U.S. Air Force TandE Days, Destin, Florida, Feb. 13-15, 2007.

${ }^{9}$ Morton, S., McDaniel, D., Dean, J., Clifton, J., and Bodkin, D., "Aircraft Stability and Control Characteristics Determined by System Identification of CFD Simulations," AIAA-2008-6378, AIAA Atmospheric Flight Mechanics Conference and Exhibit, Honolulu, Hawaii, Aug. 18-21, 2008.

${ }^{10}$ Jeans, T., McDaniel, D., Cummings, R., and Mason, W., "Aerodynamic Analysis of a Generic Fighter Using Delayed Detached-Eddy Simulation," accepted for publication in the Journal of Aircraft, 2009.

${ }^{11}$ Jeng, Y.-N., Huang, P., and Cheng, Y.-C., "Decomposition of one-dimensional waveform using iterative Gaussian diffusive filtering methods," Proceeding of the Royal Society A 464, 2009.

${ }^{12}$ Powers, S. A. and Shellenger, H. G., "The X-31 - High performance at low cost," AIAA Paper 1989-2122, AHS and ASEE Aircraft Design, Systems and Operations Conference, Seattle, WA, July 31-Aug 2, 1989, 1989.

${ }^{13}$ Ross, H. and Robinson, M., "X-31: 20 Years of Successful International Cooperation," AIAA 2003-2572, AIAA/ICAS International Air and Space Symposium and Exposition: The Next 100 Years, 14-17 July 2003, Dayton, Ohio, 2003.

${ }^{14}$ Tamrat, B., "A Post-Stall Technology (PST) Fighter Close-In-Combat Results Assessment, And A Look At New CIC Performance Evaluation Metric," AIAA Paper 2004-5173, AIAA Atmospheric Flight Mechanics Conference and Exhibit, Providence, Rhode Island, Aug. 16-19, 2004, 2004.

${ }^{15}$ Canter, D. E. and Groves, A. W., "X-31 post-stall envelope expansion and tactical utility testing," AIAA Paper 19942171, Biennial Flight Test Conference, 7th, Colorado Springs, CO, June 20-23, 1994, Technical Papers (A94-28370 09-01), Washington, DC, American Institute of Aeronautics and Astronautics, 1994, 1994.

${ }^{16}$ Alcorn, C. W., Croom, M. A., and Francis, M. S., "The X-31 experience - Aerodynamic impediments to post-stall agility," AIAA Paper 1995-362, Aerospace Sciences Meeting and Exhibit, 33rd, Reno, NV, Jan 9-12, 1995, 1995.

${ }^{17}$ Grohs, T., Fischer, B., Heinzinger, O., and Brieger, O., "X-31 VECTOR - ESTOL to the Ground Flight Test Results and Lessons Learned," AIAA Paper 2004-5029, AIAA Guidance, Navigation, and Control Conference and Exhibit, 16 - 19 August 2004, Providence, Rhode Island, 2004.

${ }^{18}$ Rohlf, D., Brieger, O., and Grohs, T., "X-31 VECTOR System Identification - Approach and Results," AIAA Paper 2004-4830, AIAA Atmospheric Flight Mechanics Conference and Exhibit, 16 - 19 August 2004, Providence, Rhode Island, 2004.

${ }^{19}$ Young, J., "X-31 VECTOR Program Summary," AIAA Paper 2004-5026,AIAA Guidance, Navigation, and Control Conference and Exhibit, Providence, Rhode Island, Aug. 16-19, 2004, 2004.

Distribution A, Approved for public release, distribution unlimited 9 of 10 
${ }^{20}$ Williams, D. L., Nelson, R. C., and Fisher, D., "An investigation of X-31 roll characteristics at high angle-of-attack through subscale model testing," AIAA Paper 1994-806, Aerospace Sciences Meeting and Exhibit, 32nd, Reno, NV, Jan 10-13, $1994,1994$.

${ }^{21}$ Rein, M., Höler, G., Schütte, A., Bergmann, A., and Löser, T., "Ground-based simulation of complex maneuvers of a delta-wing aircraf," AIAA Paper 2006-3149, 25th AIAA Aerodynamic Measurement Technology and Ground Testing Conference, 5 - 8 June 2006, San Francisco, California, 2006.

${ }^{22}$ Schütte, A., Einarsson, G., Raichle, A., Schöning, B., Orlt, M., Neumann, J., Arnold, J., Mönnich, W., and Forkert, T., "Numerical Simulation of Maneuvering Aircraft by Aerodynamic, Flight Mechanics and Structural Mechanics Coupling," AIAA Paper 2007-1070, 45th AIAA Aerospace Sciences Meeting and Exhibit, 8 - 11 January 2007, Reno, Nevada, 2007.

${ }^{23}$ Boelens, O. J., "CFD analysis of the flow around the X-31 aircraft at high angle of attack," submitted to the 27th AIAA Applied Aerodynamics Conference, 22-25 June 2009, San Antonio, TX, USA, 2009.

${ }^{24}$ Tyssel, L., "Hybrid Grid Geeneration for complex 3D Geometries," Proceedings of the 7th International Conference on Numerical Grid Generation in Computational Field Simulation, 337-346, Whistler, British Columbia, Canada, Sept. 2000.

${ }^{25}$ Tyssel, L., "The TRITET Grid Generation System," International Society of Grid Generation (ISGG), Proceedings of the 10th International Conference on Numerical Grid Generation in Computational Field Simulations, Forth, Crete, Greece, Sept. 2000.

${ }^{26}$ Cummings, R. M., Morton, S. A., and McDaniel, D. R., "Experiences in accurately predicting time-dependent flows," Progress Aerospace Sci, 2008.

${ }^{27}$ Jirásek, A. and Cummings, R. M., "Application of Volterra Functions to X-31 Aircraft Model Motion," AIAA 2009-3629, 27th AIAA Applied Aerodynamics Conference, San Antonio, Texas, June 22-25, 2009.

${ }^{28}$ Klein, V. and Morelli, E. A., Aircraft System Identification, Theory and Practise, AIAA Education Series, AIAA, Reston, Virginia, 3rd ed., 2006.

${ }^{29}$ Morelli, E. A., "System IDentification Programs for AirCraft (SIDPAC)," AIAA Paper 2002-4704, 2002.

Distribution A, Approved for public release, distribution unlimited 10 of 10 